\title{
A Common Arterial Trunk Replacing Middle Colic and Right Colic Arteries: A Case Report and Surgical Significance
}

\author{
Joseph Aziz* \\ Faculty of Health and Social Development, Unitec Institute of Technology, New Zealand
}

${ }^{*}$ Corresponding author: Joseph Aziz, Senior Lecturer of Anatomy \& Physiology, Faculty of Health and Social Development, Te-Miro Trans-disciplinary Studies -Undergraduate Department, Unitec Institute of Technology, Auckland, New Zealand, Tel: 0210642793; Email: jaziz@unitec.ac.nz

Submission: 海December 04, 2017; Published: 温 January 10, 2018

\begin{abstract}
Introduction: The mesenteric vascular supply is a combination of rich collateral networks and commonly encountered variant anatomy. The effect of normal and variant anatomy has implications on pathology, treatment choices, and planning interventions. A review of anatomic variants will assist in understanding the implications of abnormal anatomy on treatment for diseases associated with the mesentery.
\end{abstract}

Material and method: A routine dissection of mesenteric blood vessels of a 67-year old male cadaver in the dissecting room of Faculty of Medicine, Cairo University.

Results: It was noted that there was a wide caliber and long $(7.5 \mathrm{~cm})$ arterial trunk. There were absences of middle colic and right colic arteries. The middle colic artery was represented by an ascending branch while the right colic artery was represented by the descending branch.

Conclusion: The incidence of such an anomaly is relatively high. These arterial variations underscore the importance of performing vascular studies prior to major abdominal surgery.

\section{Introduction}

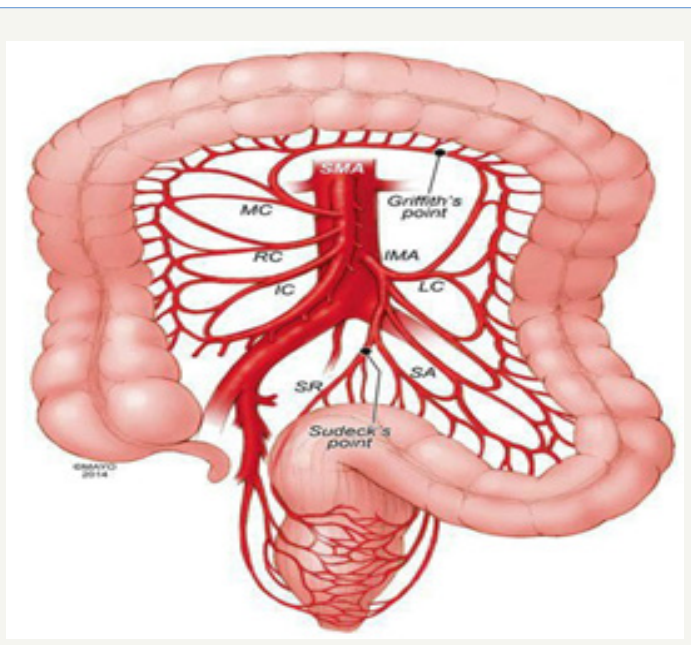

Figure 1: SMA: Superior Mesentericartery; IC: Ileocolicartery; RC: Right Colicartery; MC: Middle Colicartery.

The superior mesentericartery (SMA) is the artery of mid gut. It supplies all the derivatives of the mid gut, namely the duodenum below the opening of the bileduct, jejunum, ileum, appendix, caecum, ascending colon, right two-third soft transverse colon and the lower half of the head of the pancreas. The artery gives off several branches which include; the inferior pan creaticoduodenal artery from the posterior aspect, jejunal and ileal branches from the left surface and ileocolic, right colic and middle colic branches from right surface (Figure 1).

\section{Material and Method}

A routine dissection of the branches of the abdominal aorta particularly the mesenteric blood vessels of a 67 year-old male cadaver for teaching purposes was performed to demonstrate the branching pattern of the coeliac, SMA and IMA. The dissection was carried out in the dissecting room department of anatomy, Faculty of Medicine, Cairo University.

A large magnifying lens was used to help with dissection and to identify the branching pattern of the mesenteric arteries and to predict any abnormalities as well. A midline incision from the xiphoid process of the sternum down to the symphysis pubis was done to easily access the peritoneal cavity and to figure out the structure of its anterior compartment. The viscera were reflected after beening identified and the mesentery of the small intestine was exposed and explored at its normal original level anterior to the 3 rd part of the duodenum. The mesentery was handled carefully and opened to expose the superior mesenteric vessels. 


\section{Results}

It was noted that there was an unusual arterial trunk with wide caliber $0.2 \mathrm{~cm}$, measuring $7.5 \mathrm{~cm}$ in length, originating from the superior mesenteric artery SMA within the mesentery of the small intestine. It passed horizontally to the right where it ended just close to the ascending colon by dividing into two terminal divisions, ascending and descending The ascending branch measured $3.8 \mathrm{~cm}$ while the descending branch was little bit longer measuring $5.5 \mathrm{~cm}$. Exploration of the branches of the SMA showed absences of a separate middle colic and right colic arteries while the ileocolic artery was apparently seen in place. It was suggested that the middle colic artery was replaced by the ascending branch while the right colic artery was replaced by the descending branch (Figure 2-4).

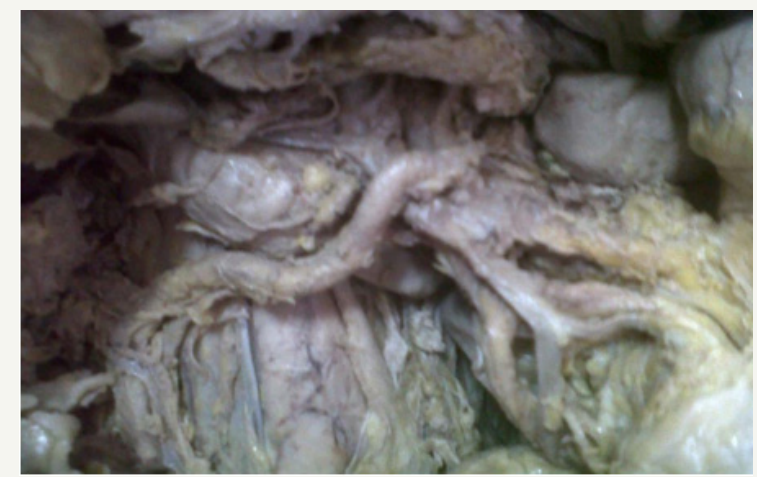

Figure 2: Arterial trunk (AT) crossing the superior mesentericvein (SMV).

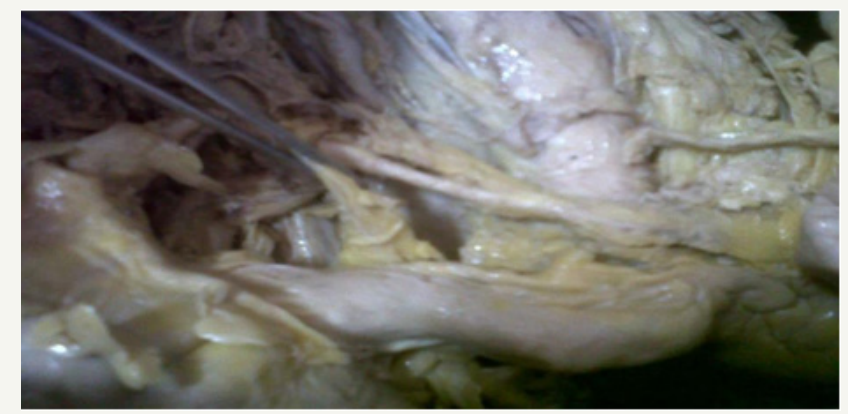

Figure 3: Descending branch $(\mathrm{DB})$ representing the right colic A.

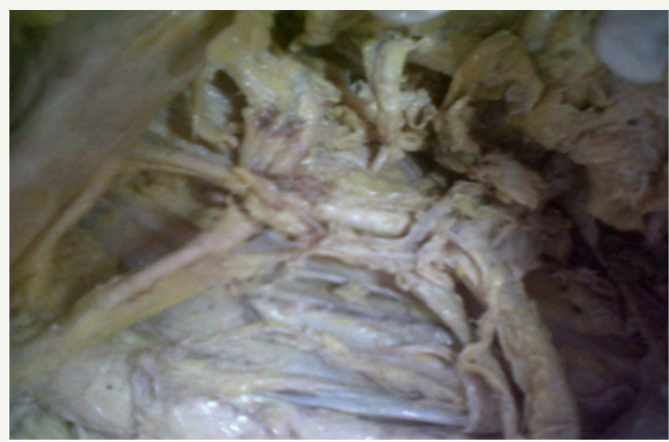

Figure 4: Ascending branch (DB) representing the Middle colic A.

\section{Discussion}

Numerous variations of the SMA regarding the origin, course and branching pattern have been reported in the literature. Ceren Gunenc and C Cem Denk reported an unusual anatomical variation of the SMA and renal artery [1]. The "normal" SMA anatomy may be present in as many as $68 \%$ of cases (Figure $5 a$ ). The ileocolic artery appears to be the most consistent structure from the SMA. The other vessels have some degree of variability. Although normally a separate branch, the middle colic artery, can share a common trunk with the right colic artery (middle colic-right colic trunk (Figure 5b) in up to $52 \%$ of cases, representing the most common variant [2-4]. If not involved in aberrant anatomy with the middle colic artery, the right colic artery may be an independent branch of the SMA (38\%) or a branch of the ileocolic artery in $8 \%$ of cases (Figure $5 c$ ). There may be an accessory right colic in 8-10\% of cases. Less commonly, the middle and right colic artery are absent $(<10 \%$ of the time), or the middle colic may send a large branch to the splenic flexure. In very rare cases, the middle colic artery may be a branch of the celiac artery [5].

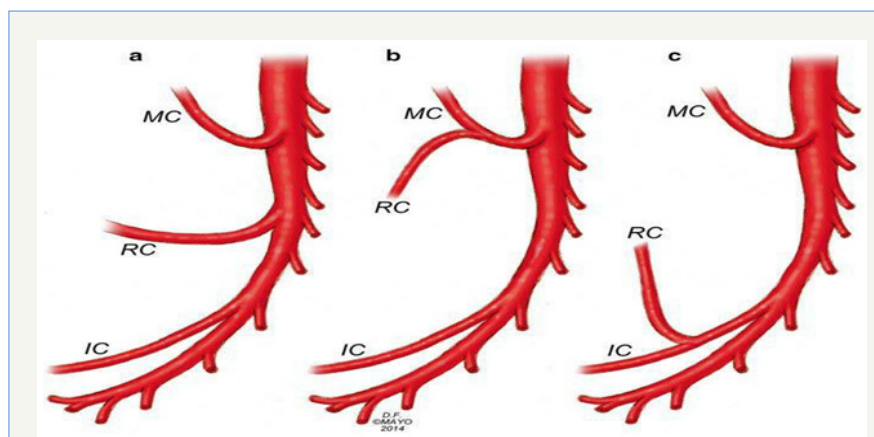

Figure 5: Superior mesenteric artery anatomic variants. IC: Ileocolic artery; RC: Right Colic artery; MC: Middle Colic artery. (a) Normal anatomy with separate IC, RC, and $\mathrm{MC}$ origins. (b) Combine $\mathrm{MC}$ and $\mathrm{RC}$ origin and separate $\mathrm{IC}$ origin. (c) Common $\mathrm{RC}$ and $\mathrm{IC}$ origin and separate $\mathrm{MC}$ origin.

\section{Conclusion}

Detailed knowledge of the different anatomical variations of the superior mesenteric artery is of extreme clinical importance, particularly, when performing laparoscopic surgery, and radiological procedures in the abdomen, and in surgical procedures such as right hemicolectomy, resection of the transverse colon and bloc resection of the head of the pancreas. The study of superior mesenteric vessels is of great importance when pancreatic head carcinoma invades the superior mesenteric vein. Also, in depth knowledge of the anomalous branching pattern of SMA is essential for the successful accomplishment of the surgical, oncologic or interventional procedures because of the large visceral territory supplied by a single vessel [6].

\section{References}

1. Günenç C, Denk CC (2006) Combined unusual anatomical variations of the superior mesenterric and right renal arteries. Clin Anat 19: 716- 717.

2. Meyers MA (1976) Griffiths' point: critical anastomosis at the splenic flexure. Significance in ischemia of the colon. Am J Roentgenol 126(1): 77-94. 
3. Geroulakos G, Cherry KJ (2002) Diseases of the visceral circulation. Oxford University Press, USA, p.228.

4. Rosenblum JD, Boyle CM, Schwartz LB (1997) The mesenteric circulation. Anatomy and physiology. Surg Clin North Am 77(2): 289-306.
5. Fisher DF, Fry WJ (1987) Collateral mesenteric circulation. Surg Gynecol Obstet 164(5): 487-492.

6. Sridhar VK, Narendra P, Vollala VR (2009) Common celiacomesenteric trunk: a rare anatomic variation. J Vasc Bras 8(3): 271-273. 